\title{
Midlife cardiorespiratory fitness and the long-term risk of chronic obstructive pulmonary disease
}

\author{
Gorm Mørk Hansen, ${ }^{1}$ Jacob Louis Marott, ${ }^{2}$ Andreas Holtermann, ${ }^{3}$ Finn Gyntelberg, ${ }^{4}$ \\ Peter Lange, ${ }_{1}^{5}$ Magnus T Jensen ${ }^{6,7}$
}

\begin{abstract}
- Additional material is published online only. To view please visit the journal online (http://dx.doi.org/10. 1136thoraxjnl-2018-212821).

For numbered affiliations see end of article.

\section{Correspondence to Dr Gorm Mørk Hansen, Herlev-Gentofte University Hospital, Medical Department 0 , Respiratory Section; Herlev- Gentofte University Hospital, Department of Cardiology, Copenhagen 2730, Denmark; gormmh@gmail.com}

Received 9 November 2018 Revised 2 May 2019

Accepted 3 May 2019 Published Online First 17 June 2019

\begin{abstract}
Background Good midlife cardiorespiratory fitness (CRF) may reduce the risk of chronic obstructive pulmonary disease (COPD). Reverse causation may play a role if follow-up time is short. We examined the association between CRF and both incident COPD and COPD mortality in employed men with up to 46 years follow-up, which allowed us to account for reverse causality.
\end{abstract}

Methods Middle-aged men $(n=4730)$ were recruited in 1970-1971. CRF was determined as VO $\mathrm{O}_{2}$ max by ergometer test. Categories of CRF (low, normal, high) were defined as \pm 1 Z-score ( \pm 1SD) above or below the age-adjusted mean. Endpoints were identified through national registers and defined as incident COPD, and death from COPD. Multi-adjusted Cox models and restricted mean survival times (RMST) were performed. Results Compared with low CRF, the estimated risk of incident COPD was $21 \%$ lower in participants with normal CRF (HR 0.79, 95\% Cl 0.63 to 0.99) and 31 $\%$ lower with high CRF (HR 0.69, 95\% Cl 0.52 to 0.91). Compared with low CRF, the risk of death from COPD was $35 \%$ lower in participants with normal CRF (HR $0.65,95 \% \mathrm{Cl} 0.46$ to 0.91 ) and $62 \%$ lower in participants with high CRF (HR $0.38,95 \% \mathrm{Cl} 0.23$ to 0.61). RMST showed a delay to incident COPD and death from COPD in the magnitude of 1.3-1.8 years in normal and high CRF vs low CRF. Test for reverse causation did not alter the results.

Conclusion In a population of healthy, middle-aged men, higher levels of CRF were associated with a lower long-term risk of incident COPD and death from COPD.

\section{INTRODUCTION}

Chronic obstructive pulmonary disease (COPD) is caused by the gradual destruction of the airways and alveoli due to inhalation of tobacco smoke, gases and particles, and is the fourth most frequent cause of death worldwide with rising incidence. ${ }^{12}$ Studies have suggested that a high level of physical activity and/or leisure time exercise is associated with a reduced risk of COPD, and that physical inactivity may accelerate disease progression. ${ }^{3}$ The terms physical activity, exercise, and physical fitness are often used interchangeably, and though the specific definitions vary, the concepts are very positively correlated. ${ }^{4}$ Several epidemiological studies with varying duration of follow-up have shown that a self-reported high level of physical activity is associated with decelerated age-related lung function decline as assessed by spirometry. ${ }^{5-9}$ However, due

\section{Key messages}

What is the key question?

- Does good midlife cardiorespiratory fitness (CRF) reduce the very long-term risk of chronic obstructive pulmonary disease (COPD)?

What is the bottom line?

- In a population of healthy, employed, Danish men followed for 46 years, we showed that good midlife CRF was associated with a reduced risk of both incident COPD and death from COPD.

Why read on?

- This study provides a novel insight into the association between CRF and the long-term risk of COPD with exceptionally long follow-up.

to the insidious and progressive nature of COPD, very long follow-up is required to minimise bias from reverse causation since subclinical disease already present at the start of follow-up may affect the measured fitness parameter. Also, self-reported physical activity and exercise is subjective and prone to overreporting, which may result in underestimation of the health benefits of a physically active lifestyle. ${ }^{10}$ In this study, with up to 46 years follow-up, we examined middle-aged, employed Danish men from the Copenhagen Male Study without COPD at baseline for the association between cardiorespiratory fitness (CRF) and the long-term risk of incident COPD and death from COPD. CRF is an objective and reproducible measurement of the maximal oxygen uptake capacity $\left(\mathrm{VO}_{2} \max \right)$, and it is strongly and consistently associated with overall level of physical fitness. ${ }^{8} 11$ The long follow-up in the present study allowed us to address the role of reverse causation by exclusion of participants who died within 10 years of the initial inclusion.

\section{METHODS}

\section{Study population}

The Copenhagen Male Study is a prospective cohort study comprised of 5286 men aged 40-59 years recruited from 14 large workplaces in Copenhagen from 1970 to 1971 . All participants completed a questionnaire, which included information on tobacco use, alcohol consumption, physical activity, level of education, current occupation, and occurrence of known diabetes mellitus. ${ }^{12-15}$ 


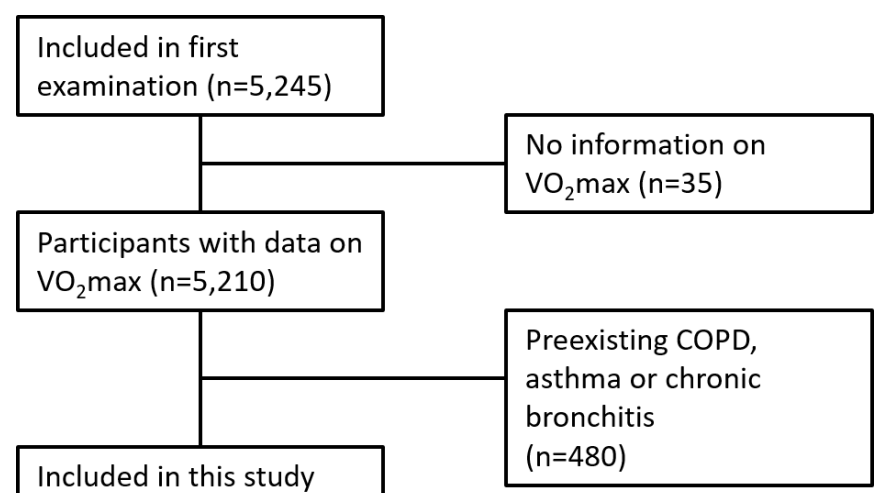

$(n=4,730)$

Figure 1 Flowchart of the Copenhagen Male Study 1970-1971. COPD, chronic obstructive pulmonary disease; $\mathrm{VO}_{2}$ max, maximal oxygen uptake capacity.

The participants classified themselves as never smokers, former smokers or active smokers. Tobacco consumption was calculated as grams per day based on the number of reported daily cigarettes, cigars, cheroots or grams of pipe tobacco. Alcohol use was registered as low, moderate or high according to average daily unit consumption. ${ }^{12-15}$ Physical activity during leisure time was self-reported as low, moderate or high. ${ }^{14}$ Socioeconomic status was based on level of education and occupation. ${ }^{16}$ Examination included interview by a physician with focus on medical history, measurement of height, weight and resting blood pressure. Participants who reported pre-existing COPD or asthma were excluded. Participants were also excluded based on the questionnaire if they responded 'yes' to the question: 'Have you experienced productive cough on most days for at least 3 months of two or more consecutive years?' (ie, the definition of chronic bronchitis). ${ }^{17}$ Figure 1 shows a flowchart of the study population.

$\mathrm{VO}_{2}$ max was estimated by standard bicycle ergometer test and Astrand's nomogram. ${ }^{18}$ The validity and reproducibility of the Astrand method is comparable to the gold standard of direct measurement of $\mathrm{O}_{2}$ consumption, however with a tendency to underestimate $\mathrm{VO}_{2}$ max. ${ }^{19} 20$

Protocols for workload (100W, $150 \mathrm{~W}$ or $200 \mathrm{~W})$ were based on the weight and height of the participant. ${ }^{12} 13$ Since $\mathrm{VO}_{2} \max$ naturally declines with age, the study population was divided into three categories of CRF defined as above or below \pm 1 Z-score $( \pm 1 \mathrm{SD})$ of the age-adjusted mean $\mathrm{VO}_{2}$ max: low CRF (bottom 15.8\%, $\mathrm{n}=745$ ), normal CRF (middle 68.6\%, $\mathrm{n}=3246$ ) and high CRF (top $15.6 \%, n=739$ ). Figure 2 shows the distribution of the three groups according to age and $\mathrm{VO}_{2}$ max.

\section{Endpoints and follow-up}

Incident COPD and death from COPD were used as endpoints. Incident COPD was defined as the first registered diagnosis of COPD using the International Classification of Diseases (ICD) codes ICD-8: 491-492, ICD-10: J41-44. Diagnoses included both outpatient and admission diagnoses, whichever occurred first. Data to 22 March 2017 were collected from the Danish National Patient Registry (DNPR) established in 1976, which covers all Danish hospitals. ${ }^{21}$ Any diagnosis of COPD in the period between the study start (1970-1971) and DNPR establishment (1976) was not registered but would be registered in later contacts after 1976. The validity of the COPD diagnosis in DNPR has previously been examined and found to be high. ${ }^{22}$

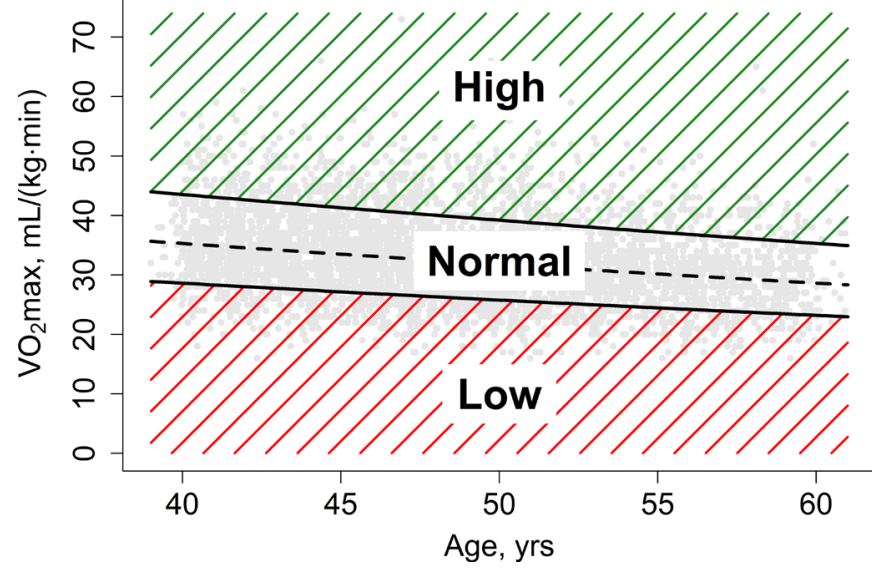

Figure 2 Cardiorespiratory fitness (CRF) groups according to age and $\mathrm{VO}_{2} \max$. The study population was divided into three categories of CRF. The categories were defined as above or below \pm 1 Z-score ( $\pm 1 \mathrm{SD}$ ) of the age-adjusted mean $\mathrm{VO}_{2} \max$ (dashed line). The bottom $15.8 \%(n=745)$ of participants were labelled low CRF, the middle $68.6 \%$ $(n=3246)$ were labelled normal CRF, and the top 15.6\% $(n=739)$ were labelled high $\mathrm{CRF}_{2} \mathrm{VO}_{2}$ max, maximal oxygen uptake capacity.

Death from COPD was identified from the Danish Register of Causes of Death to 1 January 2016 using the same ICD-codes as for incident COPD. ${ }^{23}$

Information on vital status throughout the entire duration of the study was collected from the Central Person Register (CPR) to 9 March $2017 .{ }^{24}$ Follow-up was $99.9 \%$ complete.

The assumed causal processes of the measured, and unmeasured, variables are depicted as directed acrylic graphs in online supplementary file 1 .

\section{Statistics}

Statistical analyses were performed with STATA version 12.1 (StataCorp LP, Texas, USA) and R version 3.2.0 (https://www. r-project.org/). For baseline characteristics, the categorical variables were analysed with the $\chi^{2}$-test, and the continuous variables with ANOVA. Analyses included age-adjusted and multi-adjusted Cox proportional hazards models and restricted mean survival times. For the restricted mean survival time, the full follow-up period was utilised.

Directed acrylic graphs were created using online freeware DAGitty version 2015 (http://www.dagitty.net). ${ }^{25}$

In the multivariable models, we mutually adjusted for age, body mass index (BMI) $\left(<20,20-25,>25 \mathrm{~kg} / \mathrm{m}^{2}\right)$, self-reported physical activity (low, moderate, high), smoking status (never, former, active), tobacco consumption (grams/day), alcohol consumption (0-2 units/day, 3-5 units/day, $>5$ units/day), arterial hypertension, diabetes mellitus, and socioeconomic status (high, moderate, low). The full models with variables are available in online supplementary file 2 .

The sensitivity analyses included tests for reverse causation, which were performed by excluding participants who died within the first 10 years of inclusion. Furthermore, a competing risk analysis with all causes of death as the competing event was performed. ${ }^{26} \mathrm{~A}$ p value $<0.05$ was considered significant.

\section{Ethics}

Written informed consent was obtained from all participants. Approval from an ethical committee was not possible since the 


\begin{tabular}{|c|c|c|c|c|c|}
\hline Baseline characteristics & All & Low CRF & Normal CRF & High CRF & $P$ value \\
\hline$n(\%)$ & $4730(100)$ & $745(15,8)$ & $3246(68,6)$ & $739(15,6)$ & \\
\hline Dead, all-cause, n (\%) & $4338(91.7)$ & $693(93.0)$ & $2980(91.8)$ & $665(90.0)$ & 0.10 \\
\hline Age at inclusion, years, mean (SD) & $48.7(5.3)$ & $49.1(5.2)$ & $48.6(5.4)$ & $48.9(5.3)$ & 0.10 \\
\hline CRF, mean (SD) & $33.0(7.2)$ & $23.4(2.5)$ & $32.5(4.0)$ & $44.6(5.1)$ & $<0.001$ \\
\hline \multicolumn{6}{|l|}{ Smoking, n (\%) } \\
\hline Never & $432(9.1)$ & $70(9.4)$ & $298(9.2)$ & $64(8.7)$ & \multirow[t]{3}{*}{$<0.001$} \\
\hline Former & $931(19.7)$ & $178(23.9)$ & $640(19.7)$ & $113(15.3)$ & \\
\hline Active & $3367(71.2)$ & $497(66.7)$ & $2308(71.1)$ & $562(76.0)$ & \\
\hline Grams tobacco/day, median (IQR) & $15(0-21)$ & $13(0-21)$ & $14(0-21)$ & $15(0-23)$ & $<0.001$ \\
\hline \multicolumn{6}{|l|}{ Self-reported physical activity, n (\%) } \\
\hline Low & $789(16.7)$ & $190(25.5)$ & $519(16.0)$ & $80(10.8)$ & \multirow[t]{3}{*}{$<0.001$} \\
\hline Moderate & $3428(72.5)$ & $499(67.1)$ & $2387(73.6)$ & $542(73.3)$ & \\
\hline High & $509(10.8)$ & $55(7.4)$ & $337(10.4)$ & $117(15.8)$ & \\
\hline \multicolumn{6}{|l|}{ BMI, n (\%) } \\
\hline$<20 \mathrm{~kg} / \mathrm{m}^{2}$ & $2300(48.7)$ & $237(31.9)$ & $1581(48.8)$ & $482(65.3)$ & \multirow[t]{3}{*}{$<0.001$} \\
\hline $20-25 \mathrm{~kg} / \mathrm{m}^{2}$ & $2129(45.1)$ & $395(53.1)$ & $1490(46.0)$ & $244(33.1)$ & \\
\hline$>25 \mathrm{~kg} / \mathrm{m}^{2}$ & $293(6.2)$ & $112(15.1)$ & $169(5.2)$ & $12(1.6)$ & \\
\hline Arterial hypertension, $\mathrm{n}(\%)$ & $1972(41.7)$ & $461(61.9)$ & $1332(41.0)$ & $179(24.2)$ & $<0.001$ \\
\hline Diabetes mellitus, n (\%) & $40(0.8)$ & $10(1.3)$ & $23(0.7)$ & $7(0.9)$ & 0.22 \\
\hline \multicolumn{6}{|l|}{ Alcohol consumption, n (\%) } \\
\hline Light (0-2 units/day) & $3881(82.1)$ & $568(76.2)$ & $2690(82.9)$ & $623(84.3)$ & \multirow[t]{3}{*}{$<0.001$} \\
\hline Moderate (3-5 units/day) & $701(14.8)$ & $141(18.9)$ & $459(14.1)$ & $101(13.7)$ & \\
\hline Heavy (>5 units/day) & $148(3.1)$ & $36(4.8)$ & $97(3.0)$ & $15(2.0)$ & \\
\hline \multicolumn{6}{|l|}{ Socioeconomic status, n (\%) } \\
\hline Low & $2570(54.4)$ & $406(54.6)$ & $1755(54.1)$ & $409(55.3)$ & \multirow[t]{3}{*}{0.016} \\
\hline Middle & $1349(28.6)$ & $238(32.0)$ & 919 (28.4) & $192(26.0)$ & \\
\hline High & $805(17.0)$ & $100(13.4)$ & $567(17.5)$ & $138(18.7)$ & \\
\hline
\end{tabular}

BMI, body mass index; CRF, cardiorespiratory fitness; IQR, interquartile range; SD, standard deviation.

ethics committee did not exist prior to 1980, which was after the establishment of the Copenhagen Male Study 1970-1971.

\section{RESULTS}

\section{Baseline characteristics}

Baseline characteristics of the study population are shown in table 1. Follow-up was up to 46 (mean 28.3) years. Of 4730 participants, $4338(91.7 \%)$ died. Mean age at time of study inclusion was 48.7 (SD 5.3) years. In total, 626 (13.2\%) participants were diagnosed with COPD, and 233 (4.9\%) were registered with COPD as cause of death.

A low CRF was associated with lower levels of self-reported physical activity, higher body mass index, higher alcohol consumption, lower socioeconomic status, and higher incidence of diabetes mellitus and arterial hypertension. The prevalence of former and active smokers, and the overall tobacco consumption, was higher in participants with normal and high CFR than with low CRF (table 1).

\section{Cardiorespiratory fitness and the risk of incident COPD}

Normal and high CRF was compared with low CRF in an age-adjusted Cox proportional hazard model. This showed an exposure-response relationship with reduced risk of incident COPD with increased level of CRF. The age-adjusted showed that when compared with low CRF, the risk of incident COPD was reduced by $18 \%$ in participants with normal CRF (HR 0.82 [95\% CI: 0.66 to $1.01, \mathrm{p}=0.07])$, and by $22 \%$ for participant with high CRF (HR 0.78 [95\% CI: 0.60 to $1.02, \mathrm{p}=0.08$ ]).

Figure 3 presents the results from the multi-adjusted analyses of CRF and incident COPD. The estimates were similar to those observed in the univariate analyses. The estimated risk of incident COPD was reduced by $21 \%$ in participants with

\begin{tabular}{|c|c|c|c|c|}
\hline & CRF-level & Observations, $n$ & Hazard ratio $(95 \% \mathrm{Cl})$ & $p$-value \\
\hline \multirow{3}{*}{ Incident COPD } & Low & 103 & 1 (ref.) & \\
\hline & Normal & 418 & $0.79(0.63: 0.99)$ & 0.038 \\
\hline & High & 105 & $0.69(0.52: 0.91)$ & $\longmapsto$ \\
\hline \multirow{3}{*}{ Death from COPD } & Low & 45 & 1 (ref.) & \\
\hline & Normal & 158 & $0.65(0.46-0.91)$ & 0.012 \\
\hline & High & 30 & $0.38(0.23-0.61)$ & $<0.001$ \\
\hline
\end{tabular}

Figure 3 Midlife cardiorespiratory fitness and the long-term risk of chronic obstructive pulmonary disease. The figure shows the results from the multi-adjusted Cox proportional hazards models. It displays the association between cardiorespiratory fitness (CRF) and chronic obstructive pulmonary disease (COPD) in healthy, middle-aged men. CRF is inversely associated with the long-term risk of incident COPD and death from COPD in an exposure-response relationship. 
normal CRF (HR 0.79 [95\% CI: 0.63 to $0.99, \mathrm{p}=0.038]$ ), and by $31 \%$ in participants with high CRF $(0.69$ [95\% CI: 0.52 to $0.91, \mathrm{p}=0.01])$. Cumulative incidence curves of incident COPD are available in online supplementary table 3 . The exclusion of participants who were diagnosed with COPD during the first 10 years of follow-up did not change the estimates of the sensitivity analyses (data not shown).

\section{Cardiorespiratory fitness and the risk of death from COPD}

Comparison of normal and high CRF with low CRF in an age-adjusted Cox proportional hazards model showed a reduced risk of death from COPD with increased level of CRF. The age-adjusted risk of death from COPD, relative to low CRF, was estimated to be reduced by $27 \%$ in participants with normal CRF (HR 0.73 [95\% CI: 0.52 to $1.01, \mathrm{p}=0.058]$ ), and by $50 \%$ in participants with high CRF (HR 0.50 [95\% CI: 0.32 to $0.80, p=0.003]$ ). The results from the multi-adjusted analyses of CRF and the risk of death from COPD are presented in figure 3. In this analysis the same association was observed: Compared with low CRF, the risk of death from COPD was reduced by an estimated $35 \%$ in participants with normal CRF (HR 0.65 [95\% CI: 0.46 to 0.91, $\mathrm{p}=0.012]$ ), and with $62 \%$ for participants with high CRF (HR 0.38 [95\% CI: 0.23 to $0.61, \mathrm{p}<0.001]$ ). Cumulative incidence curves of death from COPD are available in online supplementary table 3 .

\section{Restricted mean survival time}

Using restricted mean survival times, we found that increased levels of CRF were associated with a delay to both diagnosis of COPD and death from COPD. Time to diagnosis was delayed by 1.79 years for normal vs low CRF (95\% CI: 0.14 to 3.45 , $\mathrm{p}=0.034)$, and of 1.45 years $(95 \% \mathrm{CI}:-0.71-3.60, \mathrm{p}=0.19)$ for high vs low CRF. Death from COPD was delayed by 1.28 years (95\% CI: 0.04 to $2.52, \mathrm{p}=0.042$ ) for normal vs low CRF, and by 1.8 years $(95 \%$ CI 0.19 to $3.42, p=0.028)$ for high vs low CRF.

\section{Test for reverse causation}

Test for reverse causation was performed in the multi-adjusted analysis and in the restricted mean survival time-analysis. This was done by excluding all participants who died within 10 years of inclusion $(n=386)$. The results of the multi-adjusted analyses were essentially unchanged. For incident COPD the estimates were: HR 0.78 (95\% CI: 0.63 to $0.98, \mathrm{p}=0.032$ ) for normal vs low CRF, and HR 0.69 (95\% CI: 0.51 to $0.91, \mathrm{p}=0.01$ ) for high vs low CRF. For death from COPD the estimates were: HR 0.65 (95\% CI: 0.46 to $0.91, p=0.012$ ) for normal vs low CRF, and HR 0.38 (95\% CI: 0.23 to $0.61, \mathrm{p}<0.001$ ) for high vs low CRF.

The results from the restricted mean survival time-analyses after excluding all participants who died within 10 years of inclusion demonstrated even further delay to diagnosis of COPD and death from COPD: Diagnosis was delayed by 2.1 years for normal vs low CRF (95\% CI: 0.31 to $3.88, \mathrm{p}=0.021$ ), and of 1.75 years $(95 \% \mathrm{CI}:-0.56-4.06, \mathrm{p}=0.14)$ for high vs low CRF. Death from COPD was delayed by 1.47 years (95\% CI: 0.13 to $2.80, \mathrm{p}=0.032)$ for normal vs low CRF and by 2.0 years $(95 \%$ CI: 0.31 to $3.77, p=0.021$ ) for high vs low CRF.

\section{Competing risk analysis}

In a competing risk analysis with death from all causes as competing risk, normal and high CRF remained associated with a reduced risk of long-term incident COPD compared with low CRF, although the observations were not statistically significant: HR 0.86 (95\% CI: 0.69 to $1.08, p=0.19)$ for normal vs low CRF and HR 0.89 (95\% CI: 0.67 to $1.19, \mathrm{p}=0.43$ ) for high vs low CRF. Normal and high CRF remained associated with a reduced risk of death from COPD with observations that remained statistically significant: HR 0.71 (95\% CI: 0.51 to $0.99, \mathrm{p}=0.048$ ) for low vs normal CRF, and HR 0.51 (95\% CI: 0.32 to 0.83 , $\mathrm{p}<0.007)$ for low vs high CRF. Altogether, the results from the competing risk analyses suggest that the relationship between CRF, COPD and the competing event have the same direction.

\section{DISCUSSION}

In this study, we examined the association between CRF and the long-term risk of COPD in middle-aged, healthy, employed men. We found that CRF was inversely associated with the long-term risk of incident COPD and death from COPD. Compared with a low midlife CRF, participants with a high CRF had a reduced risk of incident COPD and death from COPD by approximately one third and one half, respectively. Furthermore, high midlife CRF was observed to delay time to both diagnosis and death in the magnitude of $1 \frac{1}{2}-2$ years. Test for reverse causation had no significant influence on the results. Altogether, the findings therefore point towards a possible causal inverse relationship between CRF and COPD.

To the best of our knowledge, this study is the first to report a relationship between objectively assessed CRF and the very long-term risk of COPD in healthy, middle-aged men. Through national registries, we were able to follow the participants for up to 46 years with near complete follow-up.

Our results are in line with previous studies with shorter follow-up, which have demonstrated positive associations between increased levels of physical activity and pulmonary function. 52728

In a recent Finnish study of 382 men, investigators examined $\mathrm{CRF}$ and the long-term risk of a composite endpoint defined as any diagnosis of COPD, pneumonia or asthma. ${ }^{27}$ The multi-adjusted analysis revealed an inverse, exposure-response relationship between CRF and the risk of respiratory disease when extremes of CRF-level quartiles were compared, however no separate analysis of COPD was performed, and the results were not tested for reverse causation. ${ }^{27}$ In another study, a cohort of middle-aged smokers was followed for 10 years in the Copenhagen City Heart Study. The researchers found that self-reported moderate and high levels of regular physical activity were associated with reduced lung function decline (assessed with spirometry) and a reduced risk of COPD. ${ }^{5}$ The results were later tested for time-dependent confounding in a marginal structural model which confirmed the original results. ${ }^{29}$ In another study, which followed 186 middle-aged men for 25 years, regular exercise and high levels of leisure time physical activity were associated with a slower decline in pulmonary function in all smoking categories. $^{24}$

Lung function declines gradually with age and this process is greatly accelerated in smokers. ${ }^{1}$ In the present study, smoking was very prevalent at the enrolment with more than $70 \%$ of the men being active smokers. The baseline characteristics demonstrated that smoking and the amount of tobacco consumed was higher in participants with high baseline CRF. This 'bealthy smoker effect' has previously been described in observational studies. ${ }^{30}$ The phenomenon is suggested to be related to selection bias, that is, taking up the habit of smoking may not be random and may be done more frequently by individuals who are relatively resistant to the detrimental effect of smoking. Another possible explanation on the slightly higher CRF among smokers may be a stimulating effect on 
erythropoietin level due to lower oxygen tension in the blood of smokers which stimulates the blood marrow to produce more red blood cells. Ultimately, this effect would result in an underestimation of the harmful effect of smoking demonstrated in many observational studies. ${ }^{30}$

The mechanisms that connect CRF with the development and progression of COPD are unknown. CRF is a pivotal physiological indicator of the integrated ability of the cardiac, pulmonary, muscular, and cellular systems to transport oxygen from the inspired air to the mitochondria and produce ATP as the fuel for physical activity. While no previous studies have examined the association between CRF and COPD specifically, several studies have suggested that high levels of physical activity may reduce lung function decline thereby delaying the progression of COPD. ${ }^{35-9}{ }^{30}$ We were not able to study this relationship due to lack of spirometric data in our cohort and due to our approach of using the objectively measured CRF rather than self-reported physical activity. However, other mechanisms connecting CRF and COPD are also possible. We could speculate that low-grade systemic inflammation may play a role. Physical inactivity, which is a measurable feature of even early COPD, is associated with high levels of markers of systemic inflammation. ${ }^{28}{ }^{31-33}$ This includes elevated levels of high-sensitivity C-reactive protein, fibrinogen, interleukin 1 and 6 and an increased release of reactive oxygen species from inactive muscle tissue that contribute to increased systemic oxidative stress similarly to the effects observed in smoking. ${ }^{28} 3132$ Contrarily, regular physical activity reduces both markers of systemic inflammation and oxidative stress. ${ }^{332}$ Earlier studies have shown that increased levels of inflammatory markers are related to poorer prognosis of COPD due to increased number of exacerbations, higher prevalence of comorbidities and higher mortality. ${ }^{34}$ Therefore, it is plausible that higher levels of physical activity among individuals with high levels of CRF can affect COPD development and progression.

Finally, no causality can be claimed from the observed associations which is an inherent bias of all observational studies. As such, it is possible that participants with high levels of CRF were more resilient to and less symptomatic of underlying COPD, and therefore had a delayed healthcare contact. But since death from COPD is also reduced with increasing levels of CRF, it is unlikely that delayed time to diagnosis can explain our results entirely.

Some limitations apply to the results presented in this study. Although we excluded individuals with previous diagnosis of COPD, asthma and those with symptoms of chronic bronchitis at baseline, the lung function was not assessed by spirometry at baseline and was not included in the diagnosis of incident COPD. Yet, with regard to the assessment of incident COPD, a previous study has shown a high validity of COPD diagnosis in the Danish Patient Register. ${ }^{22}$ Another limitation is lack of monitoring of smoking habits and CRF during the follow-up. However, these potential limitations would only draw the findings toward the null hypothesis and therefore underline the robustness of the findings.

\section{CONCLUSIONS}

In a population of healthy, employed, Danish men we showed that good midlife CRF was associated with a very long-term reduced risk of both incident COPD and death from COPD, and a delay to diagnosis and death. Tests for reverse causation did not change the results.
In individuals at risk of developing COPD, fitness enhancing physical activity should be encouraged not only to reduce dyspnea but also to delay development, progression and death from COPD.

\section{Author affiliations}

${ }^{1}$ Herlev-Gentofte University Hospital, Medical Department 0, Respiratory Section; Herlev-Gentofte University Hospital, Department of Cardiology, Copenhagen, Denmark

${ }^{2}$ The Copenhagen Male Study Epidemiological Research Unit, Departments of Occupational and Environmental Medicine, Bispebjerg University Hospital; The Copenhagen City Heart Study, Frederiksberg Hospital, Copenhagen, Denmark ${ }^{3}$ National Research Centre for the Working Environment; Department of Sports Science and Clinical Biomechanics, University of Southern Denmark, Copenhagen, Denmark

${ }^{4}$ The Copenhagen Male Study Epidemiological Research Unit, Departments of Occupational and Environmental Medicine, Bispebjerg University Hospital; Bispebjerg Hospital, National Research Centre for the Working Environment, Copenhagen, Denmark

${ }^{5}$ Herlev Hospital, Medical Department O, Respiratory Section; University of Copenhagen, Section of Epidemiology, Department of Public Health, Copenhagen, Denmark

${ }^{6}$ The Copenhagen Male Study Epidemiological Research Unit, Departments of Occupational and Environmental Medicine, Bispebjerg University Hospital, Copenhagen, Denmark, Copenhagen, Denmark

${ }^{7}$ Department of Cardiology, Rigshospitalet University Hospital, Copenhagen, Denmark

Acknowledgements The Copenhagen Male Study was supported by grants from King Christian X Foundation, The Danish Heart Foundation, The Danish Medical Research Council, and Else \& Mogens Wedell-Wedellsborg Foundation. Funders had no role in or influence on study design, collection and analysis of data, decision to publish, or manuscript preparation.

Contributors Conceptualisation: MTJ, GMH, FG, AH. Formal analysis: MTJ, GMH, FG, AH, JLM, PL. Methodology: MTJ, GMH, JLM. Project administration: GMH. Writing - original draft: GMH, MTJ. Writing - review \& editing: $G M H, M T J, F G, A H$, JLM, PL.

Funding This study was supported by The Danish Heart Foundation, Else \& Mogens Wedell-Wedellsborg Foundation, The Danish Medical Research Council, King Christian X Foundation.

Competing interests None declared.

Patient consent for publication Not required.

Provenance and peer review Not commissioned; externally peer reviewed.

Data sharing statement All data relevant to the study are included in the article or uploaded as supplementary information.

\section{REFERENCES}

1 Pauwels RA, Buist AS, Calverley PM, et al. Global strategy for the diagnosis, management, and prevention of chronic obstructive pulmonary disease. NHLBI/WHO global initiative for chronic obstructive lung Disease (Gold) workshop summary. Am J Respir Crit Care Med 2001;163:1256-76.

2 WHO. The top 10 causes of death. Available: http://www.who.int/mediacentre/ factsheets/fs310/en/ [Accessed 20 Oct 2018].

3 Hopkinson NS, Polkey MI. Does physical inactivity cause chronic obstructive pulmonary disease? Clin Sci 2010;118:565-72.

4 Caspersen CJ, Powell KE, Christenson GM. Physical activity, exercise, and physical fitness: definitions and distinctions for health-related research. Public Health Rep 1985; 100:126-31.

5 Garcia-Aymerich J, Lange P, Benet M, et al. Regular physical activity modifies smoking-related lung function decline and reduces risk of chronic obstructive pulmonary disease: a population-based cohort study. Am J Respir Crit Care Med 2007; 175:458-63.

6 Pelkonen M, Notkola I-L, Lakka T, et al. Delaying decline in pulmonary function with physical activity: a 25-year follow-up. Am J Respir Crit Care Med 2003;168:494-9.

7 Twisk JW, Staal BJ, Brinkman MN, et al. Tracking of lung function parameters and the longitudinal relationship with lifestyle. Eur Respir J 1998;12:627-34.

8 Cheng YJ, Macera CA, Addy CL, et al. Effects of physical activity on exercise tests and respiratory function. Br J Sports Med 2003;37:521-8.

9 Stavem K, Aaser E, Sandvik L, et al. Lung function, smoking and mortality in a 26-year follow-up of healthy middle-aged males. Eur Respir J 2005;25:618-25.

10 Brenner PS, DeLamater J, Lies DJ. Lies, damned lies, and survey Self-Reports? identity as a cause of measurement bias. Soc Psychol Q 2016;79:333-54. 
11 Jakes RWet al. Physical inactivity is associated with lower forced expiratory volume in 1 second : European Prospective Investigation into Cancer-Norfolk Prospective Population Study. Am J Epidemiol 2002;156:139-47.

12 Jensen MT, Suadicani P, Hein HO, et al. Elevated resting heart rate, physical fitness and all-cause mortality: a 16-year follow-up in the Copenhagen male study. Heart 2013;99:882-7.

13 Jensen MT, Holtermann A, Bay H, et al. Cardiorespiratory fitness and death from cancer: a 42-year follow-up from the Copenhagen male study. Br J Sports Med 2017;51:1364-9.

14 Gyntelberg F, Lauridsen L, Schubell K. Physical fitness and risk of myocardial infarction in Copenhagen males aged 40-59: a five- and seven-year follow-up study. Scand J Work Environ Health 1980;6:170-8.

15 Clausen JSR, Marott JL, Holtermann A, et al. Midlife cardiorespiratory fitness and the long-term risk of mortality: 46 years of follow-up. J Am Coll Cardiol 2018;72:987-95.

16 Svalastoga K. Prestige, class, and motility. 21. Copenhagen, Denmark: Munksgaard, 1959.

17 Smith J, Woodcock A. Cough and its importance in COPD. Int J Chron Obstruct Pulmon Dis 2006;1:305-14.

18 Åstrand P-0, Ryhming I. A nomogram for calculation of aerobic capacity (physical fitness) from pulse rate during sub-maximal work. J Appl Physiol $1954 ; 7: 218-21$.

19 Cink RE, Thomas TR. Validity of the Astrand-Ryhming nomogram for predicting maximal oxygen intake. Br J Sports Med 1981;15:182-5.

20 Macsween A. The reliability and validity of the Astrand nomogram and linear extrapolation for deriving V02max from submaximal exercise data. J Sports Med Phys Fitness 2001;41:312-7.

21 Lynge E, Sandegaard JL, Rebolj M. The Danish national patient register. Scand J Public Health 2011;39(7 Suppl):30-3.

22 Thomsen RW, Lange P, Hellquist B, et al. Validity and underrecording of diagnosis of COPD in the Danish national patient registry. Respir Med 2011;105:1063-8.
23 Helweg-Larsen K. The Danish register of causes of death. Scand J Public Health 2011;39(7 Suppl):26-9.

24 Schmidt M, Pedersen L, Sørensen HT. The Danish civil registration system as a tool in epidemiology. Eur J Epidemiol 2014;29:541-9.

25 Textor J, Hardt J, Knüppel S. DAGitty: a graphical tool for analyzing causal diagrams. Epidemiology 2011;22.

26 Fine JP, Gray RJ. A proportional hazards model for the Subdistribution of a competing risk. J Am Stat Assoc 1999;94:496-509.

27 Kunutsor SK, Laukkanen T, Laukkanen JA. Cardiorespiratory fitness is associated with reduced risk of respiratory diseases in middle-aged Caucasian men: a long-term prospective cohort study. Lung 2017;195:607-11.

28 Ertek S, Cicero A. Impact of physical activity on inflammation: effects on cardiovascular disease risk and other inflammatory conditions. Arch Med Sci 2012;8:794-804.

29 Garcia-Aymerich J, Lange P, Serra I, et al. Time-dependent confounding in the study of the effects of regular physical activity in chronic obstructive pulmonary disease: an application of the marginal structural model. Ann Epidemiol 2008;18:775-83.

30 Becklake MR, Lalloo U. The 'healthy smoker': a phenomenon of health selection? Respiration 1990;57:137-44.

31 Yanbaeva DG, Dentener MA, Creutzberg EC, et al. Systemic effects of smoking. Chest 2007;131:1557-66.

32 Laufs U, Wassmann S, Czech T, et al. Physical inactivity increases oxidative stress, endothelial dysfunction, and atherosclerosis. Arterioscler Thromb Vasc Biol 2005;25:809-14.

33 Shrikrishna D, Patel M, Tanner RJ, et al. Quadriceps wasting and physical inactivity in patients with COPD. Eur Respir J 2012;40:1115-22.

34 Miller J, Edwards LD, Agustí A, et al. Comorbidity, systemic inflammation and outcomes in the eclipse cohort. Respir Med 2013;107:1376-84. 